\title{
Hirndoping
}

\section{Selbstausbeutung per Pille}

\author{
Von Alkohol oder illegalen Drogen erhoffen sich Menschen einen Ausstieg \\ aus dem belastenden Alltag. Mittel zum Hirndoping sollen dagegen helfen, \\ sich den Anforderungen des Alltags anzupassen. Doch eher drohen Sucht und \\ Nebenwirkungen.
}

- Die Deutschen Hauptstelle für Suchtfragen (DHS) veröffentlichte am 20. Juni 2011 ein Positionspapier zum Hirndoping (www.dhs.de). Demnach wirken die zur Verbesserung ihrer geistigen Leistungsfähigkeit angewendeten Psychostimulanzien, SSRIs und Antidementiva, nicht so wie gewünscht und bergen das Risiko unerwünschter Wirkungen und einer psychischen Abhängigkeit.

\section{Akzeptanz bereits hoch}

Die Warnung kommt nicht zu früh: $80 \%$ der Oberstufen- und Berufsschüler sowie der Studierenden der Medizin, Pharmazie und Betriebswirtschaft gaben in einer Befragung an, einer leistungssteigernden und frei verfügbaren Pille ohne Nebenwirkungen positiv gegenüberzustehen [Franke AG et al. Pharma- copsychiatry 2011; 44: 60-66]. Prof. Dr. Gerd Glaeske, Bremen, betonte aber, dass die zum Hirndoping missbrauchten Arzneimittel in Studien an Gesunden keineswegs die aus der Therapie von Kranken abgeleiteten wünschenswerten Wirkungen entfalteten (siehe Tab. 1).

\section{Das Internet macht's möglich}

In den USA ist bereits ein Schwarzmarkt für die "Neuro Enhancer" entstanden. Die Beschaffung ist einfach und rezeptfrei über das Internet möglich. Juristisch und politisch ist dem wenig entgegenzusetzen. Dabei birgt das Bestellen im Internet auch noch die Gefahr, Fälschungen mit unsicherer Dosierung und gefährlichen Inhaltsstoffen zu erhalten, warnte DHS-Geschäftsführer Dr. Raphael Gassmann.
Um der Entwicklung eines Schwarzmarkts vorzubeugen, empfiehlt Glaeske eine restriktive Verordnungspraxis bei den infrage kommenden Arzneimitteln. Das Positionspapier der DHS zeigt auch Alternativen auf. Doch genügend Schlaf und Pausen, Entspannungsübungen, körperliche Aktivität, ausgewogene Ernährung oder Maßnahmen der Arbeitsplatzorganisation können langfristig die stetig wachsenden Anforderungen der Leistungsgesellschaft nur begrenzt kompensieren. Daher fordert die DHS soziale und ökonomische Rahmenbedingungen, die einem gesunden und befriedigenden Leben nicht entgegenstehen. Dazu Glaeske: „Hirndoping ist kein Weg zur Freiheit, sondern ein Mittel der Unterdrückung und der Selbstausbeutung."

FRIEDERIKE KLEIN .

- Quelle: Pressekonferenz: „Hirndoping - Die Position der Deutschen Hauptstelle für Suchtfragen", München, 20.6.2011

\section{Tabelle 1}

\section{Wirkstoffe, die zum Hirndoping missbraucht werden (Auswahl)}

\section{Wirkstoff}

Methylphenidat,

Modafinil

\section{Erhoffte Wirkung bei} Hirndoping

Steigerung der geistigen Leistungsfähigkeit (z. B. Wachheit, Aufmerksamkeit, Daueraufmerksamkeit, Konzentration)

Donepezil,

Galantamin, Rivastigmin,

Memantin

Fluoxetin, Sertralin, Citalopram, Escitalopram \section{Gedächtnisleistung} und der Lernfähigkeit

Verbesserung des psychischen Wohlbefindens (z. B. Aktivierung, Stimmungsaufhellung)
Tatsächliche Wirkung bei Gesunden

_ Schnelle psychische Abhängigkeit

- Keine Steigerung von Lerneffekten, Überschätzung der eigenen Leistungsfähigkeit

- Keine Verbesserung der kontinuierlichen Aufmerksamkeit

- Schlechtere Leistungen bei Entscheidungsaufgaben

- Verbesserung visueller Aufmerksamkeitsleistungen bei 20- bis 35-Jährigen mit schlechter Ausgangsleistung, bei guter Ausgangsleistung Verschlechterung der Leistungsfähigkeit

_ Keine Belege für die Verbesserung der Gedächtnisleistung - Häufig: Kopfschmerzen, Ruhelosigkeit, Übelkeit

Nach Informationen der DHS
Überfordert uns die Leistungsgesellschaft?

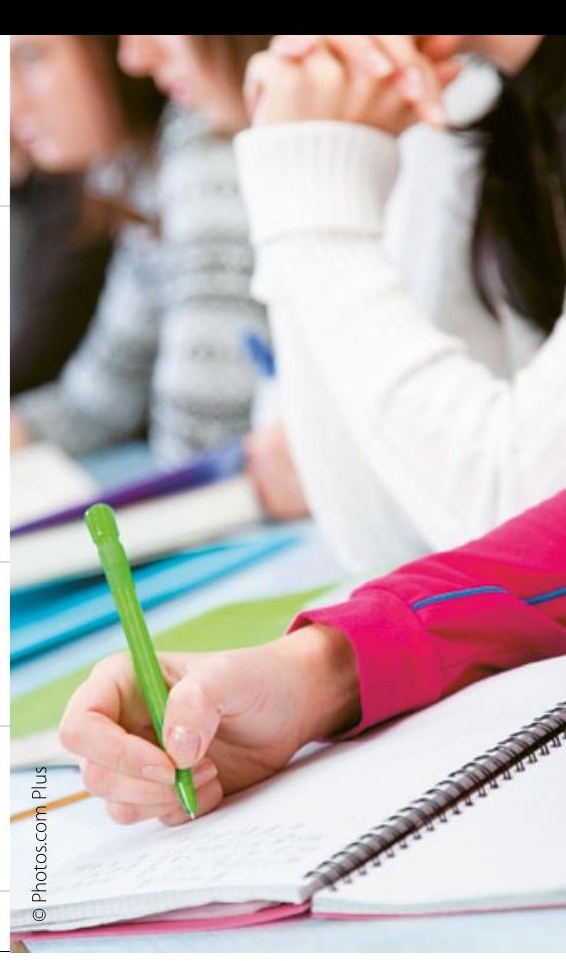

